Article

\title{
Computational Micro-Macro Analysis of Impact on Strain-Hardening Cementitious Composites (SHCC) Including Microscopic Inertia
}

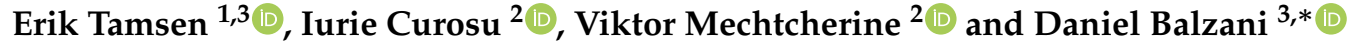 \\ 1 Institute of Mechanics and Shell Structures, TU Dresden, 01062 Dresden, Germany; \\ erik.tamsen@tu-dresden.de \\ 2 Institute of Construction Materials, TU Dresden, 01062 Dresden, Germany; iurie.curosu@tu-dresden.de; \\ mechtcherine@tu-dresden.de \\ 3 Chair of Continuum Mechanics, Ruhr University Bochum, 44801 Bochum, Germany; daniel.balzani@rub.de \\ * Correspondence: daniel.balzani@rub.de
}

Received: date; Accepted: date; Published: date

\begin{abstract}
This paper presents a numerical two-scale framework for the simulation of fiber reinforced concrete under impact loading. The numerical homogenization framework considers the full balance of linear momentum at the microscale. This allows for the study of microscopic inertia effects affecting the macroscale. After describing the ideas of the dynamic framework and the material models applied at the microscale, the experimental behavior of the fiber and the fiber-matrix bond under varying loading rates are discussed. To capture the most important features, a simplified matrix cracking and a strain rate sensitive fiber pullout model are utilized at the microscale. A split Hopkinson bar tension test is used as an example to present the capabilities of the framework to analyze different sources of dynamic behavior measured at the macroscale. The induced loading wave is studied and the influence of structural inertia on the measured signals within the simulation are verified. Further parameter studies allow the analysis of the macroscopic response resulting from the rate dependent fiber pullout as well as the direct study of the microscale inertia. Even though the material models and the microscale discretization used within this study are still simplified, the value of the numerical two-scale framework to study material behavior under impact loading is shown.
\end{abstract}

Keywords: Computational Homogenization; Impact; Microscopic Inertia; SHCC; ECC; Fiber Pullout; Rate Effect

\section{Introduction}

The characteristic composition of strain-hardening cementitious composites (SHCC) consisting of fine-grained mineral-bonded matrices in combination with high-performance polymer micro-fibers in a volume content of up to $2 \%$ is defined by a purposeful material design, accounting for the mechanical and physical properties of the cementitious matrix, of the reinforcing fibers and of their interaction, see $[1,2]$. Such a material design ensures a strain-hardening tensile behavior accompanied by the formation of multiple, fine cracks under increasing deformation. The high strain capacity prior to failure localization, notable damage tolerance and outstanding energy dissipation capacity make SHCC promising as main material for new structural elements and as strengthening layers applied on existing structures subject to earthquake, impact or blast [3].

Given the pronounced rate sensitivity of SHCC in terms of tensile strength and strain capacity, a targeted material design for applications involving dynamic loading requires a proper understanding of the governing mechanisms and phenomena at the micro- and mesoscales [4]. Furthermore, the accurate assessment of their in-situ mechanical performance in structural elements or as strengthening layers subject to dynamic loading is challenging and can be only achieved with accompanying numerical simulations, see [5]. Thus, the development of adequate, scale-linking numerical models is indispensable for assessing complex fracture phenomena in conjunction with dynamic effects as well as 
for predicting the structural performance of dynamically loaded components made of or strengthened with SHCC. A powerful tool for a simultaneous analysis of a full test specimen and the corresponding microstructure are multiscale methods. A coarse macroscopic problem uses a fine discretization of the microscale as material input. There are various frameworks that consider the dynamic effects of the microscale. Two related methods are asymptotic expansion, e.g. [6-9] which are mainly based on the original work of Bensoussan et al. [10] and the more general theory of elastodynamic homogenization by Willis [11], applied in [12-14] and others. Both methods are limited to elastic, periodic media. A more general approach is the micro-macro simulation based on a representative volume element (RVE). When the finite element method (FEM) is used on both scales in a scale-coupled manner, it is called the $\mathrm{FE}^{2}$ method. A comprehensive introduction to this theory including dynamics is given by de Souza Neto et al. [15]. Within the $\mathrm{FE}^{2}$ method there are still different approaches, usually optimized to special conditions. The framework in [6] considers a quasi-static microstructure but then applies an additional body force at the macroscale to account for microinertia effects. This framework was extended in [16] to account for localizations at the microscale under impact loading. Further FE $\mathrm{FE}^{2}$ type schemes calculate the full balance of linear momentum at the microscale. In [17] an explicit, periodic, small strain framework is presented for modeling resonant elastic metamaterials. This was extended to an implicit time integration method in [18]. By splitting the problem into a purely static and a special dynamic BVP, in $[19,20]$ the assumption of linear elasticity is used to improve the computational performance. To better capture a wider range of applied frequencies, the work of Sridhar et al. [21] uses a Floquet-Bloch transformation to build a base of eigenmodes to analyze elastic, periodic metamaterials. The framework applied in the article at hand is presented in [22]. It is also of the $\mathrm{FE}^{2}$ type, but has a more general approach. The formulations are compatible with standard FE architecture. To enable the analysis of micromechanical processes as plasticity or fiber pullout, as well as to incorporate effects of geometric nonlinearities, the framework uses a finite-strain formulation. In addition, a kinematic scale link is proposed which would allow the study of arbitrary crack paths. The framework is applicable to lower frequencies, which makes it a suitable candidate for impact investigations.

The paper at hand applies the homogenization method to study full sized SHCC specimens under impact loading, while simultaneously including the most relevant microstructural processes as matrix cracks and fiber pullout. To the best of the authors knowledge, this is the first application of a multiscale framework which considers microinertia for a simulation of fiber reinforced concrete to study the dynamic effects arising at the fine scale. The paper is organized as follows. Section 2 presents the experimental behavior of the constituents under different loading rates. Section 3 gives an overview of the dynamic $\mathrm{FE}^{2}$ framework. Section 4 discusses the material models used at the microscale. The aspects are combined in Section 5 into a simulation of a split Hopkinson tensile test, which is used to showcase the possibilities of a dynamic multiscale analysis. Finally, a conclusion is given in Section 6.

\section{Materials and Experimental Results}

The RVE presented in the paper at hand was constructed for a high-strength SHCC described in detail in previous studies by the authors Curosu and Mechtcherine [23,24]. The cementitious matrix has a fine-grained nature with aggregates consisting of a relatively small content of fine sand with particle sizes between $0.06 \mathrm{~mm}$ and $0.2 \mathrm{~mm}$. The density of the modeled SHCC is approximately $2135 \mathrm{~kg} / \mathrm{m}^{3}$ and it can vary slightly depending on the content of entrapped air [23,25]. The high-strength SHCC under investigation and the corresponding matrix material have a Young's modulus of $29 \mathrm{GPa}$, while the compressive strength ranges between $130 \mathrm{MPa}$ and $140 \mathrm{MPa}$ [23-25]. The tensile strength of the cementitious matrix under quasi-static loading is approximately 3.4 $\mathrm{MPa}$ [24].

The optimal use of high-performance polymer micro-fibers in SHCC, such as ultra-high-molecular-weight polyethylene (UHMWPE, short: PE), is justified by the criteria for strain-hardening and multiple cracking [2]. In the modeled high-strength SHCC, the PE fibers have a diameter of $20 \mu \mathrm{m}$ in average and a cut-length of $12 \mathrm{~mm}$. The nominal tensile strength as given by 
the producer is approximately $2500 \mathrm{MPa}$, the Young's modulus is $80 \mathrm{GPa}$ and the elongation at break is $3.5 \%$. The PE fibers exhibit a hydrophobic nature and no chemical adhesion to cementitious matrices [25]. Their bond is of frictional and mechanical nature, whereas their longitudinally grooved surface profile ensures additionally a mechanical interlock [4]. With such surface properties the PE fibers exhibit a controlled pullout behavior under constant interfacial shear stress [25].

Since these fibers exhibit a pronounced non-linear tensile behavior and rate dependent tensile strength, Young's modulus and elongation capacity, extensive micromechanical investigations were performed to characterize their tensile behavior in form of stress-strain relationships in an amplified piezoelectric actuator at displacement rates ranging from $0.005 \mathrm{~mm} / \mathrm{s}$ to $50 \mathrm{~mm} / \mathrm{s}$ (strain rates between $0.001 \mathrm{~s}^{-1}$ and $\left.1 \mathrm{~s}^{-1}\right)$. The free length of the fibers in the tension experiments was $5 \mathrm{~mm}$. The fibers were glued at the top end to the loading frame and at the bottom end to the force sensor [4,23]. The results showed an increase in tensile strength and Young's modulus, but a decrease in elongation capacity at higher displacement rates, see Figure 1. It was assumed that the rate sensitivity of the Young's modulus of the fibers determined the pronounced rate dependency of the fiber-matrix bond properties as well. This was demonstrated in single-fiber pullout experiments with a fiber-embedment length of $2 \mathrm{~mm}$, see Figure 2.

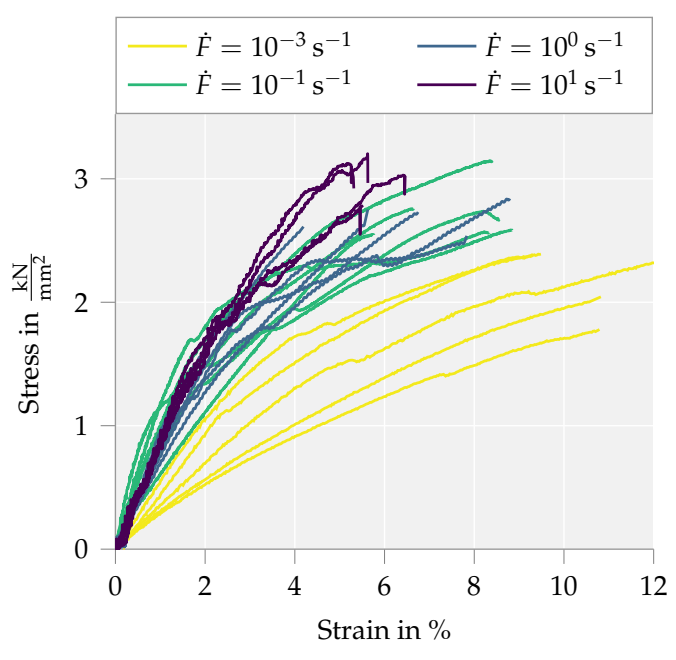

Figure 1. Results of PE fiber tension tests at different strain rates, data from $[4,23]$.

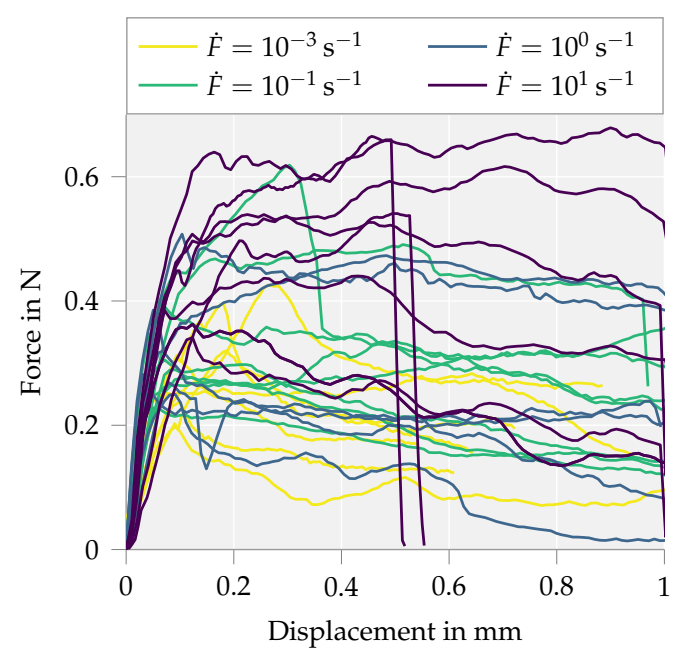

Figure 2. Results of PE fiber in pullout tests at different displacement/loading rates, data from [4, 23].

The pullout tests were performed in the same testing setup under identical displacement rates as the single-fiber tension tests. For details regarding specimen preparation and testing configuration see [4]. Assuming a rate independent Poisson's ratio, the dynamically enhanced Young's modulus limited the radial contraction of the loaded fibers and, consequently, the reduction in interfacial confinement during pullout. Note that the stroke in the testing device was limited to $1 \mathrm{~mm}$ and the fibers could not be pulled out from the matrix specimens completely. The entire pullout pattern for this fiber-matrix combination under quasi-static loading can be found in [25].

Both the single-fiber tension and pullout experiments served as experimental basis for the calibration of the micromechanical parameters in the developed numerical model. Due to the limitations imposed by the testing facilities and measuring techniques, the micromechanical experiments assumed displacement rates considerably lower than the crack opening speeds in SHCC subject to tensile impact loading in Hopkinson bar tests [4,26], the latter serving for validation purposes in the numerical study presented in the paper at hand. Thus, for the modeling of SHCC, the derived micromechanical parameters at higher strain/displacement rates were extrapolated assuming a linear rate dependency. Whether this assumption matches the real rate dependency can be investigated in a numerical parameter study, as presented in the next sections. 


\section{Numerical Two-Scale Framework Accounting for Microscopic Inertia}

This section provides an overview of the assumptions the applied numerical multiscale framework is based on and presents the resulting formulations necessary for an implementation of the homogenization scheme. A more detailed description of the full derivation is given in [22]. The proposed framework is an $\mathrm{FE}^{2}$ homogenization method with the key characteristic that it considers the full balance of linear momentum at the microscale. This enables the direct analysis of full dynamic fields at the microscale, while simultaneously allowing the study of resulting effects at the macroscale. Depicted is the two-way coupling, where each macroscopic integration point is associated with a separate microscopic RVE simulation. The macroscopic values are required as input for the microscopic boundary value problem (BVP). By using appropriate averaging relations and kinematic links, a consistent scale bridging for dynamic loading is established. After solving for microscopic equilibrium, the homogenized fields of stress $\overline{\boldsymbol{P}}$ (here in terms of the first Piola-Kirchhoff stress tensor) and the homogenized inertia force vector $\bar{f}^{\rho}$ are passed to the macroscopic problem, along with four essential tangent moduli $\overline{\mathbb{A}}^{P, F}, \overline{\mathbb{A}}^{P, u}, \overline{\mathbb{A}}^{f, F}$ and $\overline{\mathbb{A}}^{f, u}$, representing the derivatives of stress and body force with respect to deformation gradient $\overline{\boldsymbol{F}}$ and displacement $\bar{u}$, respectively. In the following, values associated with the macroscale are indicated by a bar $\boldsymbol{-}$. In order to enable the analysis of versatile micromechanical phenomena, a finite-strain formulation is used. The (undeformed) reference and the (deformed) current configuration are linked by the displacement $u=x-X$, where $X \in \mathcal{B}$ refers to the coordinates in the undeformed reference configuration and $x \in \mathcal{S}$ to the coordinates in the deformed configuration. The transformation between the configurations in terms of vector elements is described by the deformation and displacement gradients, respectively $\boldsymbol{F}=\partial_{X} \boldsymbol{x}=\mathbf{1}+\boldsymbol{H}$ and $\boldsymbol{H}=\partial_{X} \boldsymbol{u}$, such that $\boldsymbol{x}=\boldsymbol{F X}$. To simplify the notation, the origin of the microscopic coordinates is chosen as the geometrical center of the RVE, with $\int_{\mathcal{B}} \boldsymbol{X} \mathrm{d} V=\mathbf{0}$. This choice has no influence on the results. Under the assumption of scale separation, the microscopic deformation $x$ can be split into a sum of terms,

$$
x=\bar{u}+\bar{F} X+\widetilde{u}
$$

Two terms result directly from the macroscale: a constant part $\bar{u}$, which describes the macroscopic rigid body translations, and a homogeneous part $\overline{\boldsymbol{F}} \boldsymbol{X}$, defined in terms of the macroscopic deformation gradient. $\widetilde{u}$ denotes the microscopic displacement fluctuation field, which is the field the microscopic BVP is solved for. Analogously, the microscopic deformation gradient can be written as

$$
\boldsymbol{F}=\overline{\boldsymbol{F}}+\widetilde{\boldsymbol{H}} \quad \text { with } \quad \widetilde{\boldsymbol{H}}=\partial_{X} \widetilde{\boldsymbol{u}} .
$$

To account for the microscale dynamics, an extended version of the Hill-Mandel condition of macro homogeneity also called the Principle of Multiscale Virtual Power is adopted, as base for the derivation of the framework, c.f. [27] and [15]. It ensures that the virtual work of the macroscale coincides with its respective microscopic volume average. The resulting averaging equation for the effective macroscopic stress $\overline{\boldsymbol{P}}$ and the effective macroscopic body force vector $\bar{f}$ are given as

$$
\overline{\boldsymbol{P}}=\langle\boldsymbol{P}-\boldsymbol{f} \otimes \boldsymbol{X}\rangle \quad \text { and } \quad \bar{f}=\langle f\rangle .
$$

Herein, $\langle\bullet\rangle=\frac{1}{V} \int_{\mathcal{B}} \bullet \mathrm{d} V$ is an abbreviation for the volume average of a microscopic quantity. A principal ingredient of the Hill-Mandel condition, is the assumption of a clear separation of scales. This stipulates that the fluctuations of mechanical fields at the microscale need to be significantly smaller than those of the macroscopic problem. For dynamic homogenization this signifies in practice, that additionally the principal wavelength of the applied macroscopic loading must be sufficiently larger than the size of the RVE. For dealing with time derivatives, the Newmark method [28] is applied, a 
widely used implicit numerical time integration method of first order. First, the microscopic element formulations are viewed in more detail. Subsequently the respective macroscopic element equations are given.

\subsection{The Microscopic Problem}

To allow a full dynamic analysis at the microscale, the microscopic balance of linear momentum is given by

$$
\operatorname{Div} \boldsymbol{P}+f=\mathbf{0}
$$

This paper models impact loading, where gravitational forces are negligible compared to the inertia forces. Therefore, only the inertia part of the body forces $f^{\rho}$ is considered here. The relevant body force vector is defined as $f:=f^{\rho}=-\rho_{0} \ddot{u}$ with $\rho_{0}$ denoting the density of the microscale components in the undeformed configuration. Following the standard FEM algorithm, the global tangent stiffness matrix $\widehat{K}$ is assembled from the element matrix,

$$
\begin{gathered}
\widehat{\boldsymbol{k}}^{e}=\boldsymbol{k}^{e}+\frac{1}{\beta \Delta t^{2}} \boldsymbol{m}^{e}, \text { with } \\
\boldsymbol{k}^{e}=\int_{\mathcal{B}^{e}} \boldsymbol{B}^{e^{\mathrm{T}}} \mathbb{A} \boldsymbol{B}^{e} \mathrm{~d} V \text { and } \boldsymbol{m}^{e}=\int_{\mathcal{B}^{e}} \boldsymbol{N}^{e} \rho_{0} \boldsymbol{N}^{e^{\mathrm{T}}} \mathrm{d} V,
\end{gathered}
$$

where $\boldsymbol{N}^{e}$ is the classical element matrix of shape functions, $\boldsymbol{B}^{e}$ denotes the B-matrix containing the derivatives of the shape functions, and $\mathbb{A}$ is the matrix representation of the material tangent modulus, defined as $\mathbb{A}=\partial_{\boldsymbol{F}} \boldsymbol{P} . \beta$ is one of the two Newmark parameters. Throughout this work the parameters are set as $\beta=0.25$ and $\gamma=0.5$. Analogously to the stiffness matrix, the global residuum matrix $\widehat{R}$ is obtained by the assembly of the element-wise counterparts in matrix representation,

$$
\widehat{\boldsymbol{r}}^{e}=\int_{\mathcal{B}^{e}}\left(\boldsymbol{B}^{e^{\mathrm{T}}} \boldsymbol{P}+\boldsymbol{N}^{e} \rho_{0} \ddot{\boldsymbol{u}}\right) \mathrm{d} V .
$$

After including Dirichlet boundary conditions, the resulting discrete system of equations at the microscale reads

$$
\widehat{K} \Delta \widetilde{D}=\widehat{R} .
$$

The macroscopic displacements and deformation gradient and their time derivatives are used to define boundary conditions on the RVE, c.f. (1). To ensure a consistent application of the macroscopic values, certain constraints need to be enforced at the microscale. The first kinematic link concerns the deformation gradient, $\overline{\boldsymbol{F}}=\langle\boldsymbol{F}\rangle$. It postulates that the volume average of the microscopic deformation gradient must equal the deformation gradient at the macroscale. This is prescribed by applying periodic boundary conditions on the RVE. To enable a dynamic framework, it needs to be ensured that no arbitrary rigid body motions are possible. Within this work a simple solution is used for the second kinematic constraint. By applying the macroscopic displacements $\bar{u}$ to the corners of the RVE and simultaneously forcing the microscopic displacement fluctuations to be zero, a direct coupling of the macroscopic displacements is achieved. This constraint is chosen a priori and might not be optimal for all problems, however for the problem at hand it is well suited. A softer and more general constraint which applies the macroscopic displacements as the volume average using Lagrange multipliers is presented in [22]. 


\subsection{The Macroscopic Problem}

After identifying the formulations at the microscale based on macroscopic values, the bilateral coupling is realized by defining the respective macroscopic values depending on the microscopic fields, c.f. (3). In the same way as for the microscale, the complete macroscopic balance of linear momentum including inertia is considered. Here the weak form of linear momentum is given without the contributions of external traction as,

$$
\bar{G}:=\int_{\overline{\mathcal{B}}} \delta \overline{\boldsymbol{F}}: \overline{\boldsymbol{P}} \mathrm{d} V+\int_{\overline{\mathcal{B}}} \delta \overline{\boldsymbol{u}}^{\mathrm{T}} \overline{\boldsymbol{f}}^{\rho} \mathrm{d} V=0
$$

Once more, only body forces related to inertia are regarded, such that $\bar{f}:=\bar{f}^{\rho}=\left\langle f^{\rho}\right\rangle$. To apply the standard Newton-Raphson scheme, the linearized balance of linear momentum is obtained as

$$
\operatorname{Lin} \bar{G}=\bar{G}+\Delta \bar{G}=0 \quad \text { with } \quad \Delta \bar{G}=\int_{\overline{\mathcal{B}}} \delta \overline{\boldsymbol{F}}: \Delta \overline{\boldsymbol{P}} \mathrm{d} V+\int_{\overline{\mathcal{B}}} \delta \overline{\boldsymbol{u}}^{\mathrm{T}} \Delta \overline{\boldsymbol{f}}^{\rho} \mathrm{d} V
$$

Within the multiscale framework the macroscopic values of stress $\overline{\boldsymbol{P}}$ and inertia $\bar{f}^{\rho}$ are defined in terms of the microscale. Furthermore, the microscopic fields depend on both the macroscopic acceleration $\ddot{\bar{u}}$ as well as the macroscopic deformation gradient $\overline{\boldsymbol{F}}$. Therefore, the effective macroscopic stress and inertia for any given point at the macroscale are sensitive to both its displacement as well as the respective deformation gradient. This is a special property arising from the consideration of the microscale dynamics. From this observation it follows directly that the linearized terms are expanded as

$$
\Delta \overline{\boldsymbol{P}}=\frac{\partial \overline{\boldsymbol{P}}}{\partial \overline{\boldsymbol{F}}}: \Delta \overline{\boldsymbol{F}}+\frac{\partial \overline{\boldsymbol{P}}}{\partial \ddot{\bar{u}}} \cdot \Delta \ddot{\bar{u}} \quad \text { and } \quad \Delta \overline{\boldsymbol{f}}^{\rho}=\frac{\partial \overline{\boldsymbol{f}}^{\rho}}{\partial \overline{\boldsymbol{F}}}: \Delta \overline{\boldsymbol{F}}+\frac{\partial \overline{\boldsymbol{f}}^{\rho}}{\partial \ddot{\overline{\boldsymbol{u}}}} \cdot \Delta \ddot{\overline{\boldsymbol{u}}}
$$

The four emerging sensitivities are defined as

$$
\overline{\mathbb{A}}^{P, F}=\partial_{\overline{\boldsymbol{F}}} \overline{\boldsymbol{P}}, \quad \overline{\mathbb{A}}^{P, u}=\partial_{\ddot{\bar{u}}} \overline{\boldsymbol{P}}, \quad \overline{\mathbb{A}}^{f, F}=\partial_{\overline{\boldsymbol{F}}} \bar{f}^{\rho} \quad \text { and } \quad \overline{\mathbb{A}}^{f, u}=\partial_{\ddot{\bar{u}}} \bar{f}^{\rho}
$$

By applying standard FE discretization to the linearized weak form (10) while considering (11), the macroscopic element stiffness matrix $\widehat{\bar{k}}^{e}$ and the element residuum vector $\widehat{\bar{r}}^{e}$ are identified. Here the matrix representation of the moduli in index notation is used, where lowercase indices refer to the spacial dimension $n_{\mathrm{dm}}$, and uppercase indices to the total degrees of freedom of an element $n_{\text {edf }}$. This yields the definition of the full macroscopic element matrices as

$$
\begin{aligned}
\widehat{\bar{k}}_{P Q}^{e}= & \int_{\mathcal{B}^{e}}\left(\bar{B}_{i j P}^{e} \overline{\mathbb{A}}_{i j m n}^{P, F} \bar{B}_{m n Q}^{e}+\frac{1}{\bar{\beta} \Delta t^{2}} \bar{B}_{i j P}^{e} \overline{\mathbb{A}}_{i j k}^{P, u} \bar{N}_{Q k}^{e}\right. \\
& \left.+\bar{N}_{P i}^{e} \overline{\mathbb{A}}_{i m n}^{f, F} \bar{B}_{m n Q}^{e}+\frac{1}{\bar{\beta} \Delta t^{2}} \bar{N}_{P i}^{e} \overline{\mathbb{A}}_{i k}^{f, u} \bar{N}_{Q k}^{e}\right) \mathrm{d} V \text { and } \\
\hat{\bar{r}}_{P}^{e}= & \int_{\mathcal{B}^{e}}\left(\bar{B}_{i j P}^{e} \bar{P}_{i j}+\bar{N}_{P i}^{e} \bar{f}_{i}^{\rho}\right) \mathrm{d} V .
\end{aligned}
$$

By inserting the averaging equations into the definitions of the moduli and evaluating the linearized weak form of the microscale at equilibrium, the four closed form expressions can be identified in terms of the microscopic fields. This allows an efficient numerical algorithm. The macroscopic tangent moduli have been presented in [22,29]. 


\section{Micromechanical Material Models}

To present the capability of the dynamic multiscale framework of analyzing microstructures under impact loading, micromechanical models must be implemented that display the most important characteristics. For SHCC, this involves the modeling of the matrix, including a possibility to include cracks as well as the implementation of a fiber pullout mechanism.

\subsection{SHCC Matrix}

As the relevant loading considered within this work is tension, the complex behavior of the matrix under compression can currently be neglected. Therefore the elastic Neo-Hooke material law is used for the matrix material. The Neo-Hookean constitutive law yields a simple expression to model elastic material behavior at large strains. The first Piola-Kirchhoff stress tensor is given as

$$
\boldsymbol{P}=(\lambda \ln [\operatorname{det}[\boldsymbol{F}]]-\mu) \boldsymbol{F}^{-\mathrm{T}}+\mu \boldsymbol{F},
$$

with the Lamé constants $\lambda$ and $\mu$. For more details see e.g. Bonet and Wood [30]. In the tensile regime, the crack development is the most important mechanical mechanism of the matrix. The simulation of proper crack propagation is a highly complex field and not in the scope of the current work. As an approximation, a simple erosion technique is implemented for the matrix. Once the specified matrix material reaches a stress threshold $\sigma_{\mathrm{cr}}$ in loading direction, its stiffness is reduced to a small value $E_{\mathrm{cr}}$, resulting in an effective crack. As the threshold is evaluated at the local material point, this method is mesh dependent. Therefore only simulations using the same microscopic mesh will be directly compared. Due to the approximation of the fibers as truss elements directly connected to the matrix nodes, this erosion method can not be applied evenly to all matrix elements, as artificial stress localization at the shared nodes would lead to a non-physical erosion of the fiber anchorages. Consequently the crack location needs to be selected before the computation.

\subsection{Effective Fiber Pullout}

The full fiber pullout is represented by a linear truss element. The effective material model consists of a general 1D Neo-Hookean material law with two additional features. The model includes a strain rate sensitivity, as observed in the micromechanical test of the fiber material and a damage formulation to represent the pullout behavior, c.f. Section 2. It is based on the simplified assumption that the fibers are engaged by a crack crossing it in the center, leading to the same pullout function for all fibers. The standard 3D Neo-Hookean stress formulation can be simplified to 1D as

$$
P=\frac{1}{2} E\left(F-\frac{1}{F}\right)
$$

where $E$ is the Young's modulus. This is then extended with a multiplicative approach to include a damage and strain rate formulation as

$$
\widehat{P}=P(1+\Omega)(1-D) .
$$

Here, $\Omega$ denotes the dynamic increase, which takes on only positive values. It is defined using a logarithmic function of the rate of the deformation gradient, as

$$
\Omega= \begin{cases}\alpha^{\mathrm{I}} \ln \left[\frac{\dot{F}}{\alpha^{\mathrm{II}}}\right] & \dot{F} \geq \alpha^{\mathrm{II}} \\ 0 & \dot{F}<\alpha^{\mathrm{II}}\end{cases}
$$

The two parameters $\alpha^{\mathrm{I}}$ and $\alpha^{\mathrm{II}}$ respectively determine the slope and zero value of the logarithmic function. This allows an increase in stress for high deformation rates. The damage formulation is the 
governing mechanism to represent the effective fiber pullout. The scalar parameter $D$ takes on values of 0 to 1 , where 1 represents a fully damaged state, in this case a full fiber pullout. Applying the strain equivalence principle, an exponential damage function has been chosen as

$$
D=D_{\infty}\left(1-\exp \left(-\left(\frac{\psi_{\mathrm{D}}}{D_{\text {rate }}}\right)^{D_{\text {shape }}}\right)\right) .
$$

The damage value $D$ is determined based on the internal variable $\psi_{\mathrm{D}}$, representing the effective energy considered for damage.

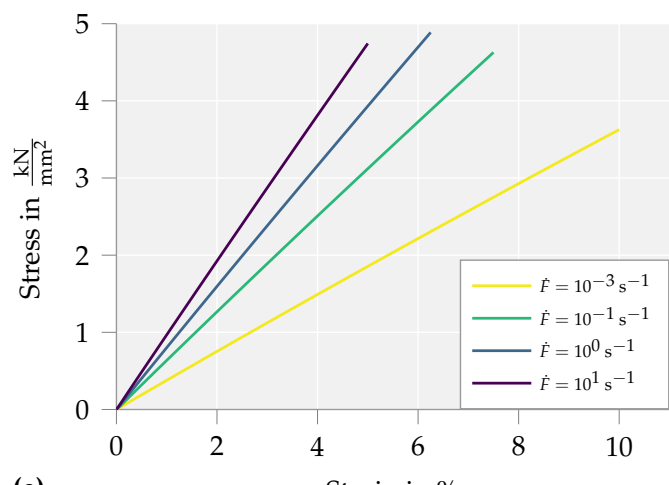

(a)

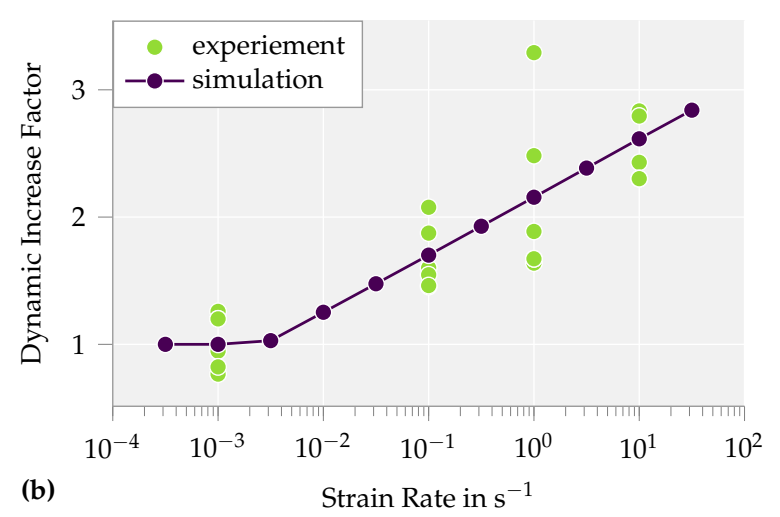

Figure 3. Simulation of single-fiber tension test with rate-dependent material properties, (a) stress-strain diagram, (b) the dynamic increase factor of the secant stiffness at fracture compared to experimental results from [4]. Material parameters used: $E=50 \mathrm{kN} / \mathrm{mm}^{2}, \alpha^{\mathrm{I}}=0.19$ and $\alpha^{\mathrm{II}}=1.8 \cdot 10^{-3}$.

It is defined as the maximum value of the strain energy function $\psi_{0}$ which has so far been reached. Thus, the damage evolves only when $\psi_{0}>\psi_{\mathrm{D}}$. This results in a discontinuous damage approach. There are three material parameters associated with the damage formulation. $D_{\infty}$ defines the maximum reachable damage value. The model parameter $D_{\text {rate }}>0$ influences the velocity of the damage evolution, $D_{\text {shape }}$ enables the modification of the overall shape of the function, where values below 1 will increase the damage rate at the beginning, while decreasing it for larger deformations.

First we analyze the strain rate effect without the damage formulation, modeling a simple dynamic fiber tension test, as presented in Section 2. Figure 3a presents the stress strain curve of the the 1D Neo-Hookean material for different strain rates. To compare the simulation to the experiment, the secant modulus at maximum strain is plotted against the applied strain rate. The linear increase in stress plotted on a logarithmic scale is clearly visible. 


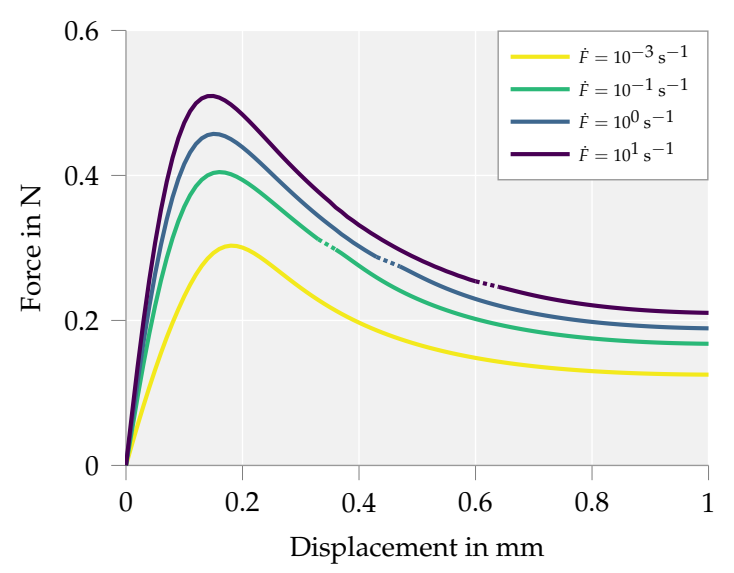

Figure 4. Simulation of single-fiber pullout test for different strain rates. Material parameters free length: $E=50 \mathrm{kN} / \mathrm{mm}^{2}, \alpha^{\mathrm{I}}=0.19$ and $\alpha^{\mathrm{II}}=1.8 \cdot 10^{-3}$. Material parameters embedded fiber: $E=300 \mathrm{kN} / \mathrm{mm}^{2}, \alpha^{\mathrm{I}}=0.08, \alpha^{\mathrm{II}}=1.8 \cdot 10^{-3}, D_{\infty}=0.998, D_{\text {rate }}=2.0$ and $D_{\text {shape }}=0.2$.

Secondly, the damage formulation is activated and fitted to the presented single-fiber pullout tests in Figure 2. The simulation uses two elements, one representing the free length of the fiber where only the rate sensitivity is active and the second element represents the embedded part of the fiber where the pullout takes place. Here both the strain rate sensitivity as well as the damage is active. The stress-strain curves for four different strain rates, applied at the boundary are given in Figure 4 . Comparing this to the experimental results, shows that the overall phases of debonding and pullout are captured. The experiments under higher strain rates show a gradual shift from slip-softening to slip-hardening, see Figure 2. This phenomenon has not been included in the model.

\section{Numerical Examples}

The last sections described the multiscale framework as well as the material models created for the microscale simulation. This section combines the two by using a well known experimental setup as example problem: the split Hopkinson bar tension test. First, the experimental setup and results are discussed. Then a quasi static calculation is used to calibrate the numerical material parameters. Finally, the full split Hopkinson bar simulation is presented and used to show the possibilities of the multiscale framework to perform dynamic simulations.

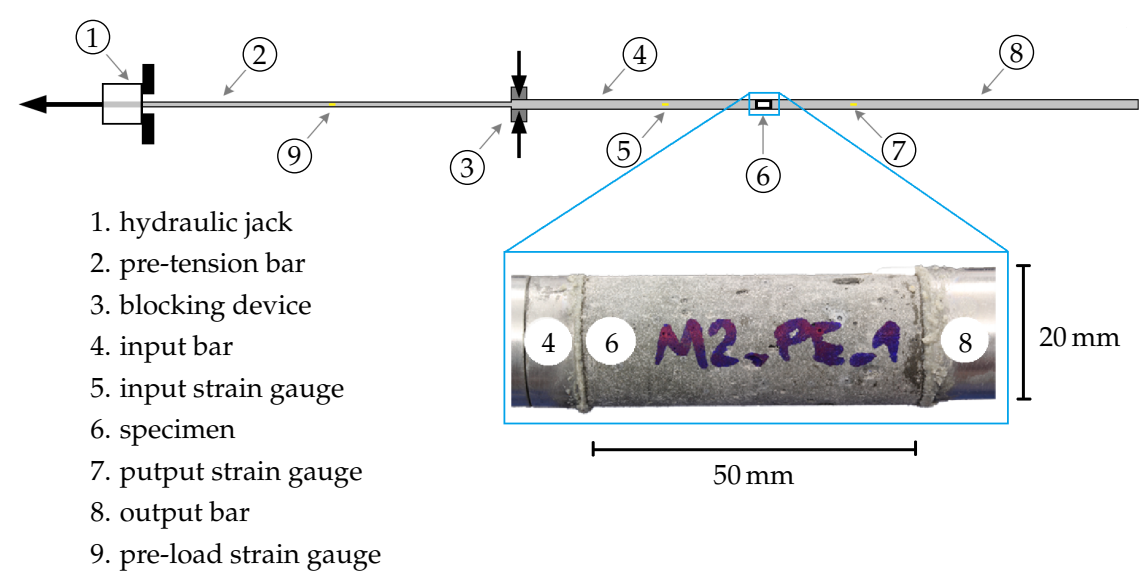

Figure 5. Split Hopkinson tension bar setup, based on [4,24]. 


\subsection{Split Hopkinson Bar Experiment}

In the split Hopkinson tension bar setup [24], the specimen is sandwiched between two aluminum bars having front and end surface contact, as visualized in Figure 5. The split Hopkinson tension bar setup consists of an input bar of length $3 \mathrm{~m}$ and of an output bar $6 \mathrm{~m}$ long, both bars made of aluminum and having a diameter of $20 \mathrm{~mm}$. A high-strength steel bar $6 \mathrm{~m}$ long having a diameter of $12 \mathrm{~mm}$ used as a pre-tensioned bar for generating the loading pulse of trapezoidal shape of $2.4 \mathrm{~ms}$ duration and with a rise-time of about $60 \mu \mathrm{s}$, see Figure 6.

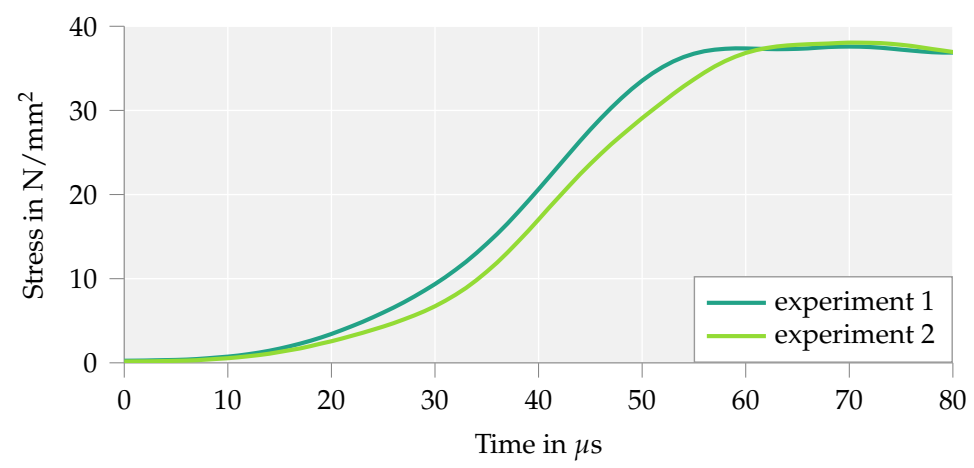

Figure 6. The characteristic trapezoidal loading pulse of the modified split Hopkinson tension bar, as measured in the in input bar, from $[4,24]$. Here only the rise is depicted a the failure occurs before the plateau is reached.

The maximum displacement speed in the test was $6 \mathrm{~m} / \mathrm{s}$ and the specimen length was $50 \mathrm{~mm}$, which ensured a peak strain rate of $120 \mathrm{~s}^{-1}$. The application of the elastic, uniaxial stress wave propagation theory to the Hopkinson bar system [31] allows calculation of the forces and and the displacements and acting on the two faces of the specimen in contact with the input and output bars, respectively. From this the stress at the two interfaces can be inferred, denoted as $\sigma_{1}$ and $\sigma_{2}$ within this work. The specimen is assumed to reach dynamic stress equilibrium if the force-time response at both ends (derived in the input and output bar) approach each other. This condition imposes a certain number of wave reverberations inside the specimen before damage initiation (cracking) and it is essential for an accurate derivation of the stress-strain relationships. With a specimen length of only $50 \mathrm{~mm}$ in the presented experiments, the dynamic stress equilibrium is reached before first crack formation.

The resulting stress-strain curves are compared to quasi-static measurements in Figure 7.

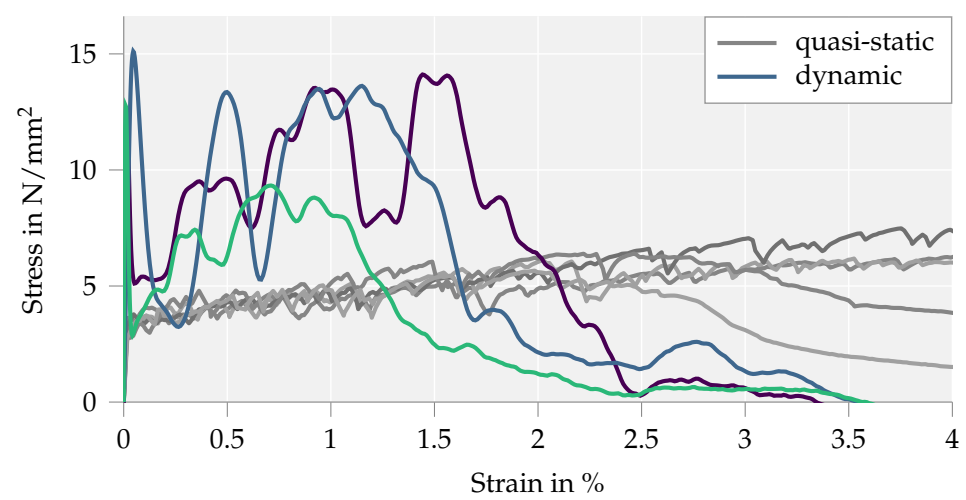

Figure 7. Experimental stress-strain curves for a split Hopkinson bar tension test with SHCC, data from $[4,24]$. The quasi-static results are given in gray as a comparison. 
Damage initiation in the matrix substantially reduces the stiffness of the specimen and the effective strain rate in the matrix. This explains the high initial stress peak and the subsequent multiple cracking occurring at lower stress levels. Moreover, the formation of cracks causes additional wave reflections in the sample, leading to pronounced oscillations of the captured waves in both input and output bars, and resulting in an unsteadiness of the derived stress-strain curves, as shown in Figure 7.

Furthermore, the dynamic tensile curves in the SHTB exhibit higher stresses but lower strains at failure localization, this representing the characteristic rate sensitivity of the high-strength SHCC. The rate-induced reduction of strain capacity can be traced back to a considerably less pronounced multiple cracking compared to quasi-static conditions and is a result of an exaggerated dynamic enhancement of the fiber-matrix bond compared to that of the fiber tensile strength. Such unbalanced micromechanical rate sensitivities lead to a shift from fiber pullout to fiber rupture at higher strain rates, which is disadvantageous with regard to steady strain-hardening and multiple cracking. This phenomenon cannot be reproduced by the simplified numerical model currently presented in the paper at hand.

\subsection{Quasi-Static Simulation}

Considering the simplified numerical models discussed in Section 4, there is no gain using a complex microstructure. Processes that would benefit from a detailed microstructure with a realistic distribution and number of fibers, as e.g. crack evolution or a fiber pullout behavior depending on the angle to the fracture face are not within the scope of this paper. Therefore, it is sufficient to discretize the microstructure as a single fiber intersected by a single crack embedded in a cubic RVE with an edge length of $1 \mathrm{~mm}$. The RVE is depicted within Figure 8a.
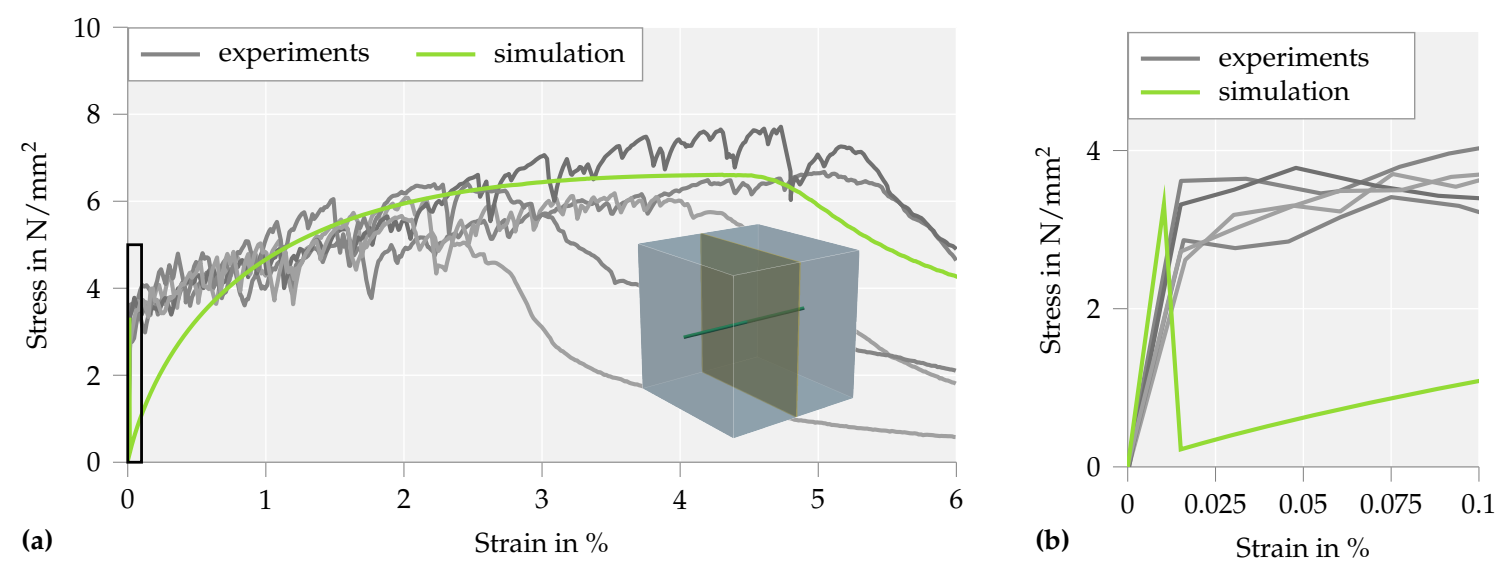

Figure 8. Results of the quasi-static multiscale simulation, compared to the experimental data. Part (a) shows the loading up to $6 \%$ strain and depicts the selected RVE. Part (b) shows a zoomed in detail of part (a), focusing on the cracking of the matrix in the RVEs. Experimental data from [4,24].

The mesh consists of three quadratic brick elements, two for the matrix and the one in the center with the possibility for cracking. The embedded fiber is simulated by a single truss element in loading direction. Unfortunately, the micromechanical measurements presented in the last section can not be directly extrapolated to the case of fully embedded fibers. Therefore, the two-scale SHCC simulation is calibrated by using the results of a quasi-static tension test. To replicate the quasi-static tension experiment, a multiscale simulation is used which consists of five truss elements at the macroscale. The bar is fixed at one end and a linear displacement load is applied at the other. The stress is recorded at the boundary and the is strain computed as the boundary displacement divided by the specimen length. The following material parameters are applied: for the matrix $E=29 \mathrm{kN} / \mathrm{mm}^{2}$ and $v=0.3$, additionally for the crack $E_{\mathrm{cr}}=10^{-3} \mathrm{kN} / \mathrm{mm}^{2}$ and $\sigma_{\mathrm{cr}}=5 \mathrm{kN} / \mathrm{mm}^{2}$ and for the fiber $E=40 \mathrm{kN} / \mathrm{mm}^{2}$, $A=0.18 \mathrm{~mm}^{2}, D_{\infty}=0.9982, D_{\text {shape }}=0.36$ and $D_{\text {rate }}=0.2$. The resulting stress-strain curve is 
compared to the experiment in Figure 8, including a zoomed-in section up to the first crack. The overall fit is good, however instead of a gradual strain hardening behavior a sharp drop in stress is observed in the simulation once the tensile strength $\sigma_{\mathrm{cr}}$ is reached. This phenomenon is due to the fact that in a quasi-static setting a homogeneous stress state is obtained at the macroscale. Thus, as there is no natural variation in material parameters, all RVEs fracture simultaneously. Nevertheless, after the cracking of the matrix, i.e. when the fibers are engaged, the general debonding behavior matches well that observed in the experiment.

\subsection{Split Hopkinson Bar Simulation}

To properly capture the essential details, first the experimental setup needs to be replicated. A sketch of the macroscopic BVP is given in Figure 9. It consists of a row of truss elements, discretized in $10 \mathrm{~mm}$ sections.

Macroscale

(a)

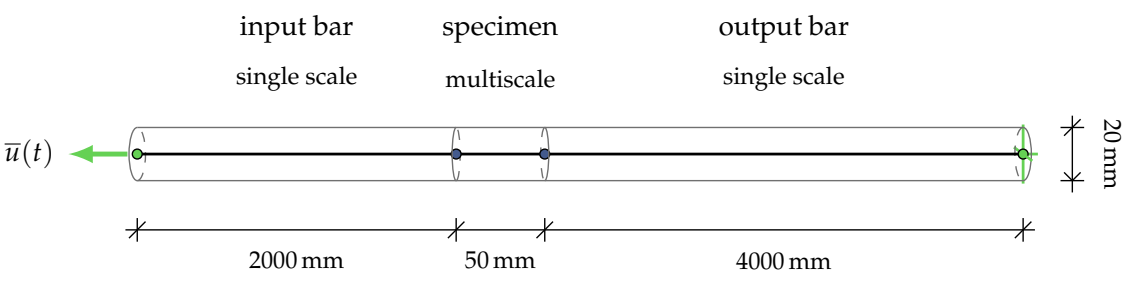

Microscale

Figure 9. Schematic visualization of the boundary value problem representing the split Hopkinson tension test. Part (a) depicts the macroscopic problem, part (b) shows the discretization of the SHCC microstructure applied in the multiscale simulation of the test specimen.

The input and output bars are simulated with standard single scale elements, whereas the two-scale homogenization framework is used for the SHCC specimen to include the simplified RVEs at the microscale. The next step is the choice of the input load, which is applied via a displacement boundary. Using the measured signal from the experiments, a piece-wise polynomial function, formulated to represent the loading conditions. It consists of three parts

$$
\begin{aligned}
\bar{u}^{\mathrm{I}}(t) & =\frac{14}{275} t v_{\mathrm{c}}\left(\frac{2 t}{t_{\mathrm{vc}}}\right)^{3}, \\
\bar{u}^{\mathrm{II}}(t) & =\frac{t v_{\mathrm{c}}}{275}\left[7\left(\frac{2 t}{t_{\mathrm{vc}}}\right)^{8}-12\left(\frac{2 t}{t_{\mathrm{vc}}}\right)^{7}+16\left(\frac{2 t}{t_{\mathrm{vc}}}\right)^{6}+19-\frac{34}{3}\left(\frac{2 t}{t_{\mathrm{vc}}}\right)^{-1}\right] \text { and } \\
\bar{u}^{\mathrm{III}}(t) & =v_{\mathrm{c}}\left(t-\frac{529}{825} t_{\mathrm{vc}}\right) .
\end{aligned}
$$

The transitions between the respective functions are at $\bar{u}^{\mathrm{I}}\left(0.592 t_{\mathrm{vc}}\right)=\bar{u}^{\mathrm{II}}\left(0.592 t_{\mathrm{vc}}\right)$ and $\bar{u}^{\mathrm{II}}\left(t_{\mathrm{vc}}\right)=$ $\bar{u}^{\mathrm{III}}\left(t_{\mathrm{vc}}\right)$, so that the loading function is defined as

$$
\bar{u}^{\mathrm{BC}}(t)= \begin{cases}\bar{u}^{\mathrm{I}}(t) & 0 \leq t \leq 0.592 t_{\mathrm{vc}} \\ \bar{u}^{\mathrm{II}}(t) & 0.592 t_{\mathrm{vc}}<t \leq t_{\mathrm{vc}} . \\ \bar{u}^{\mathrm{III}}(t) & t>t_{\mathrm{vc}}\end{cases}
$$

Figure 10 compares the chosen displacement function $\bar{u}^{\mathrm{BC}}$ and the time derivatives, velocity and acceleration, to two experimental measurements. 


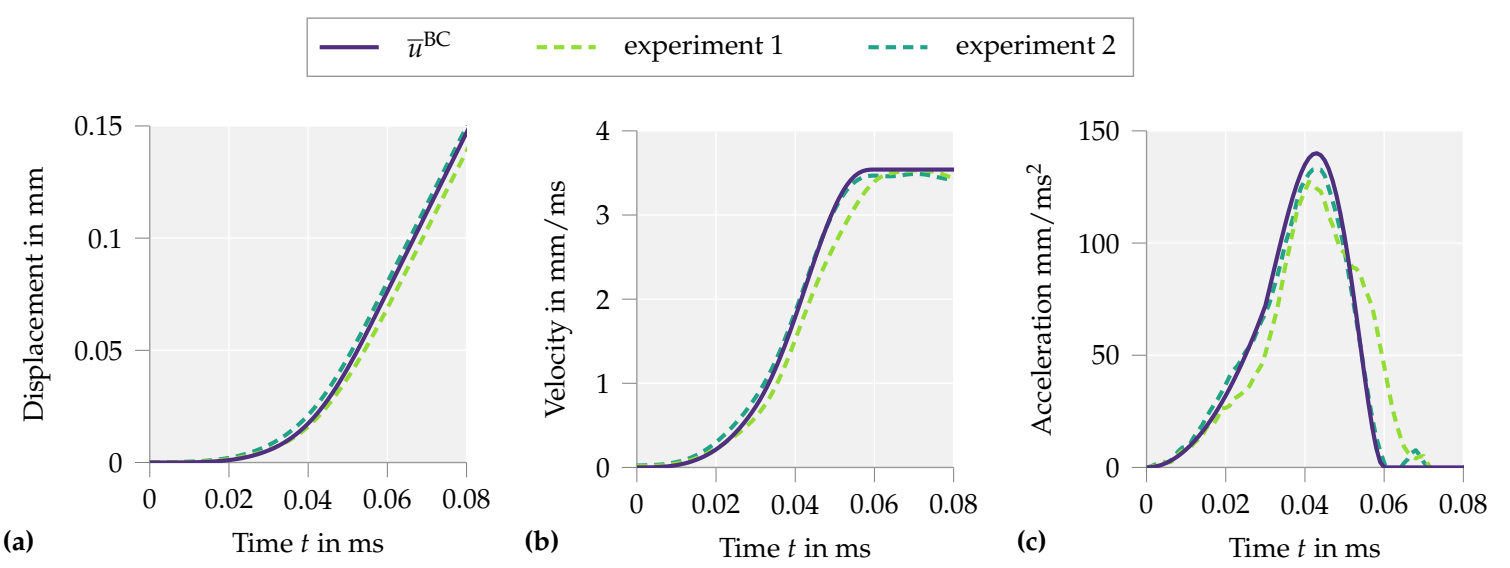

Figure 10. Loading function $\bar{u}^{\mathrm{BC}}(23)$ and its first two time derivatives, compared to the two experiments from [4], with $t_{\mathrm{vc}}=60 \mu \mathrm{s}$ and $v_{\mathrm{c}}=3540 \mathrm{~mm} / \mathrm{s}$.

Here the significance of the two loading parameters is visible. The first parameter $t_{\mathrm{vc}}$ defines the time when the transition from the acceleration phase to the phase of constant velocity is completed. The second parameter $v_{\mathrm{c}}$ sets the constant velocity. These parameters are easily identified from the experimental data. Even though the experimental data could have been used as a direct input at the boundary, the function has the advantage that parameter studies can be easily conducted to analyze the influence of the loading conditions on the specimen's response. In addition to the adjusted material parameters used in the quasi-static calculation, further parameters accounting for the dynamics must be selected. The same dynamic material parameters as identified for the fiber pullout experiments are assumed: for the matrix and the crack $\rho_{0}=2100 \mathrm{~kg} / \mathrm{m}^{3}$, and for the fiber $\rho_{0}=980 \mathrm{~kg} / \mathrm{m}^{3}, \alpha^{\mathrm{I}}=0.08$ and $\alpha^{\mathrm{II}}=0.51$. However, the values of $\alpha^{\mathrm{I}}$ and $\alpha^{\mathrm{II}}$ should only be viewed as a rough approximation, as the experiments do not represent the same conditions as in the fully embedded fibers. In addition, the applied strain rate during the split Hopkinson bar experiment is significantly higher then during the fiber tension and fiber pullout tests. Nevertheless qualitative parameter studies can be conducted.

The first results of the split Hopkinson bar simulation are presented in Figure 11.

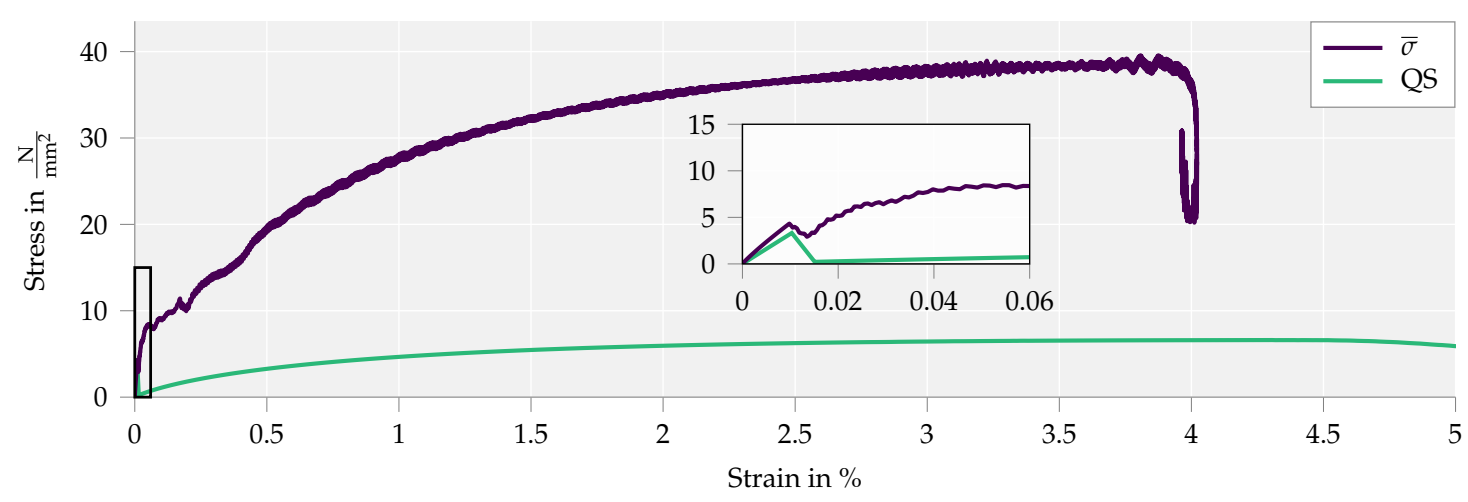

Figure 11. Results of the split Hopkinson bar simulation. The average of the stress-strain signals at both interfaces $(\bar{\sigma})$ compared to the quasi-static computation (QS). The zoom shows the initial cracking of the matrix.

It compares the dynamic stress-strain curve to the quasi static results. The increase in stress level due to the dynamic conditions is evident. In addition, a shift is observed from the instantaneous fracture of all RVEs in the quasi-static case to a successive multiple cracking behavior, as also observed in the experiments. To further understand the results and showcase the utility of the numerical framework, four parameter studies are conducted. Firstly, the two loading parameters are varied to 
understand the general influence of the loading conditions. Secondly, the influence of the strain rate sensitivity of the effective fiber pullout model is studied. Finally, the effect of the microscale inertia on the macroscopic measurements is presented.

\subsubsection{Parameter Study $-t_{\mathrm{cv}}$}

To study the influence of the applied loading function, the parameter $t_{\mathrm{vc}}$ is varied. A smaller value $t_{\mathrm{vc}}$ represents a faster rise time. This entails a higher acceleration. Therefore, this parameter allows to visualize the influence of the initial acceleration on the measured signal, while the second parameter, the value of constant velocity $v_{c}$ remains unchanged. To better understand the effects, the two signals $\bar{\sigma}_{1}$ and $\bar{\sigma}_{2}$ are each analyzed in a separate plot, see Figure 12.

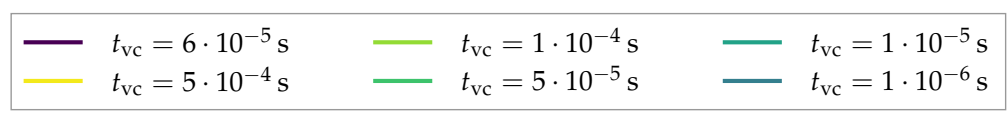

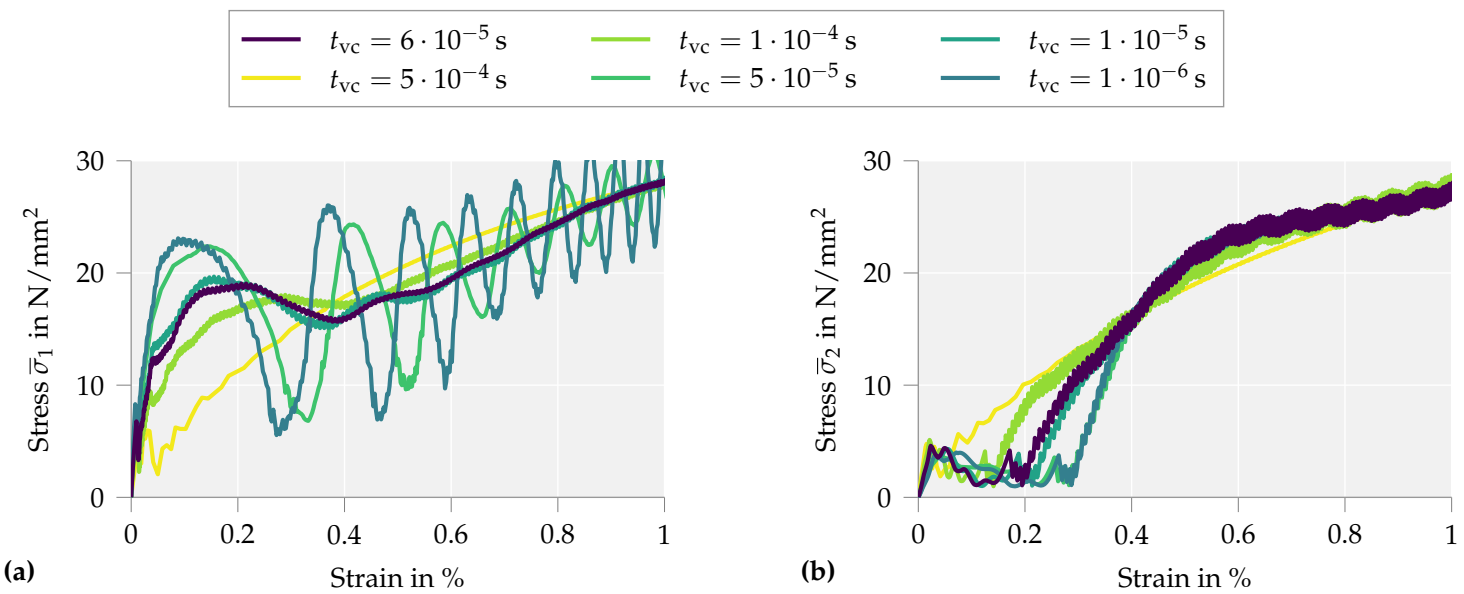

(a)

Figure 12. Analysis of the variation in rise time $t_{\mathrm{cv}}$ from $5 \cdot 10^{-4} \mathrm{~s}$ to $10^{-6} \mathrm{~s}$, with $v_{\mathrm{c}}=3540 \mathrm{~mm} / \mathrm{s}$. Plot (a) depicts the signal $\bar{\sigma}_{1}$ at the input face and (b) the respective signal $\bar{\sigma}_{2}$ at the output face.

With increasing acceleration, i.e. a shorter rise time $t_{\mathrm{vc}}$, the initial peak at the input face increases, as well as the subsequent macroscopic stress fluctuations. The only noticeable difference at the output face is a slight delay in stress increase for faster applied loads. This apparent delay is a simple result of the analyzed properties, as for a constant wave speed through the specimen the wave front will reach the output face at larger overall strains when the load is applied faster.

\subsubsection{Parameter Study $-v_{\mathrm{cv}}$}

The other loading parameter is $v_{c}$. It controls the constant velocity during the impact wave. Increasing the velocity leads to a higher stress level during the loading pulse.

$\begin{array}{ll}v_{\mathrm{c}}=3540 \mathrm{~mm} / \mathrm{s} \\ v_{\mathrm{c}}=2000 \mathrm{~mm} / \mathrm{s}\end{array}-\begin{aligned} & v_{\mathrm{c}}=3000 \mathrm{~mm} / \mathrm{s} \\ & v_{\mathrm{c}}=7080 \mathrm{~mm} / \mathrm{s}\end{aligned} \quad v_{\mathrm{c}}=14160 \mathrm{~mm} / \mathrm{s}$
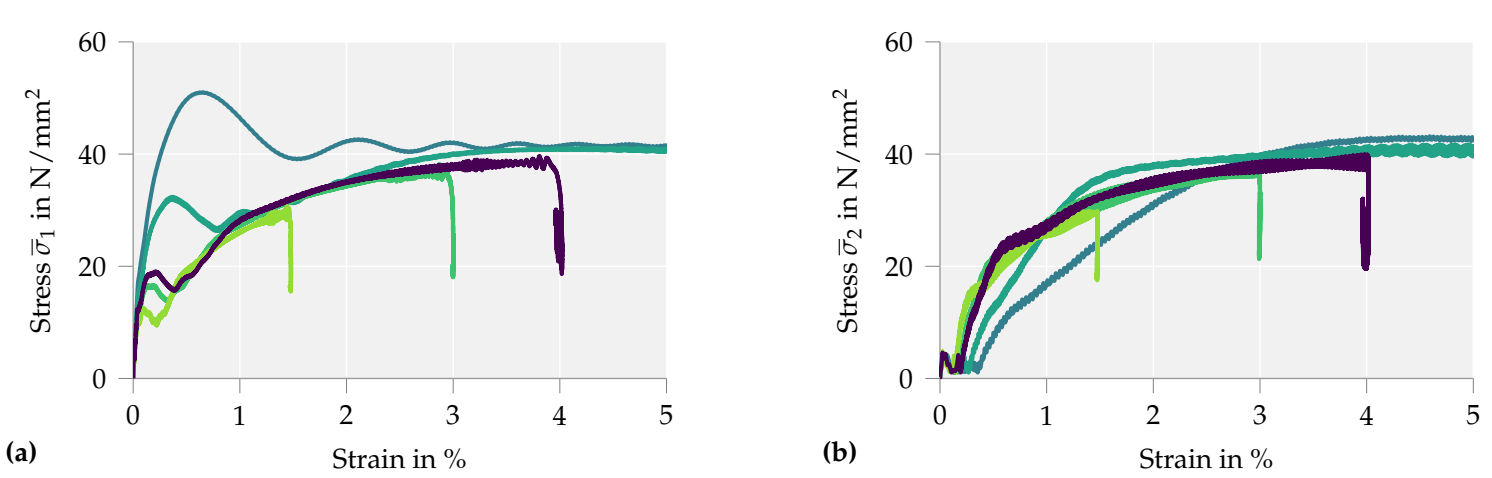

Figure 13. Analysis of the variation of $v_{\mathrm{c}}$ from $2000 \mathrm{~mm} / \mathrm{s}$ to $14160 \mathrm{~mm} / \mathrm{s}$, with $t_{\mathrm{cv}}=6 \cdot 10^{-5} \mathrm{~s}$. Plot (a) depicts the signal $\bar{\sigma}_{1}$ at the input face and (b) the respective signal $\bar{\sigma}_{2}$ at the output face . 
In addition to an increase in strain rate, the maximum acceleration during the initial phase increases, as the elevated speed is reached in the same time frame. The resulting stress-strain curves are depicted in Figure 13. The two effects discussed in the previous parameter study on $t_{\mathrm{cv}}$ are again observed. However, varying $v_{c}$ changes not only the initial loading phase, but also the overall stress-strain curve at the loading face. Therefore, the first stress peak observed in Figure 13a is a combined result of the strain-rate sensitivity of the fibers and the macroscopic inertia. In addition, with increasing $v_{\mathrm{c}}$ the stress equilibrium is reached only at higher strain rates. Another effect concerns the drop in stress at the end of the curves. This is not a global failure but rather the result of a decrease in strain rate, as will be visible in the subsequent analysis. This parameter study demonstrates that, with a reduction in the rise time of the loading wave, the condition of dynamic stress equilibrium in the sample is violated and an experimental derivation of the material response based on the one-dimensional wave theory is not accurate.

\subsubsection{Parameter Study - Strain-Rate Sensitivity of the Fiber}

After investigating the influence of the loading conditions on the obtained stress-strain curves, the dynamic influence of the microscale simulation on the macroscopic response is analyzed. First, the strain-rate sensitivity of the fiber is regarded. The results of the variation of $\alpha^{\mathrm{I}}$ is given in Figure 14.

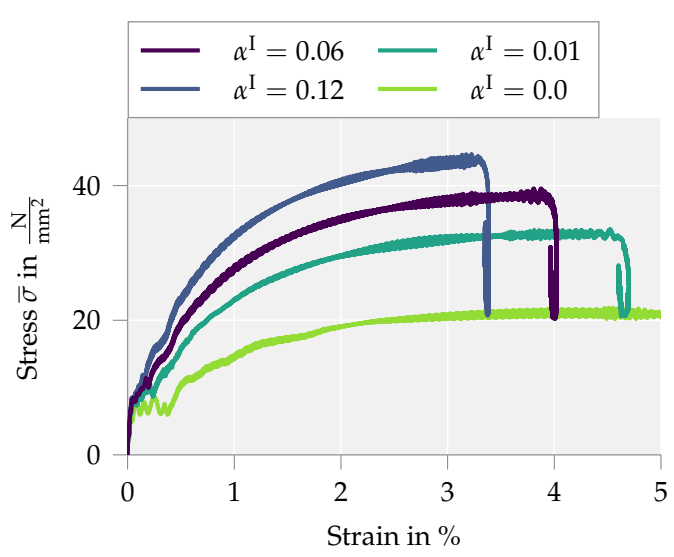

Figure 14. Analysis of the variation of the parameter $\alpha^{\mathrm{I}}$ from 0 to 0.16 .

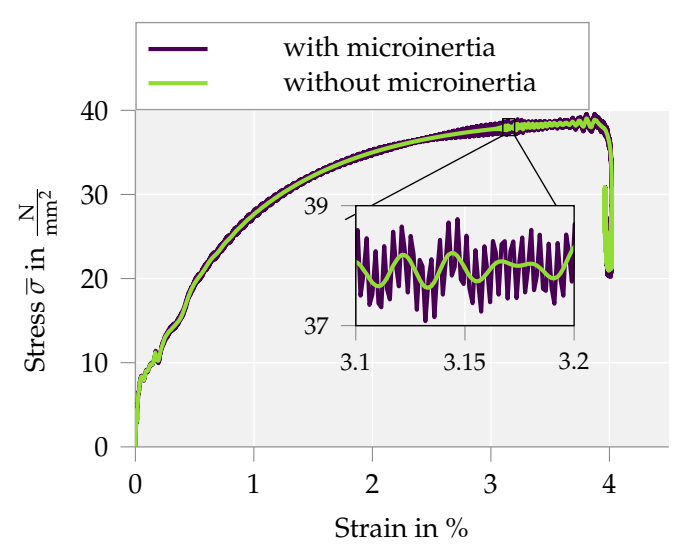

Figure 15. Analysis of the influence of microinertia on the macroscopic response.

Here the stress average $\bar{\sigma}$ of the two recorded stress signals is shown. As expected, with increasing strain-rate sensitivity of the fibers the macroscopic stress level increases as well. From this analysis it is evident that the previously observed drop in stress is due to a reduction in strain rate. This effect is due to a phase of a quasi-rigid translation of the specimen within the system, where the overall deformation does not change, but at the same time the strain rate decreases. For higher values of $\alpha^{\mathrm{I}}$ the fibers are effectively stiffer, resulting in a higher wave speed though the specimen. Therefore, the phase of quasi-rigid translation is reached earlier.

\subsubsection{Parameter Study - Microinertia}

Finally, the simulation is run without considering the inertia at the microscale compared to the full dynamic simulation as presented in Figure 11. The average of the measured stress at the specimen interfaces is given in Figure 15, including a zoomed section to highlight the difference. For the presented microstructure the overall macroscopic behavior does not appear to be significantly influenced by microscale inertia effects. This is not surprising, as the chosen RVE combined with the microscopic material models does only allow for moderate dynamic activity. High frequency stress oscillations arising at the microscale once the crack has been formed are the results of the microcracks being able to freely open and close, as the fiber is anchored at the RVE boundary. 


\section{Summary and Conclusion}

The influence of dynamic loading during micromechanical experimental tests was analyzed. After an introduction of the applied numerical homogenization framework for dynamic analysis, simplified material models were presented and calibrated with respect to the experimental data at hand. Finally, the two parts were combined in a multiscale simulation of a split Hopkinson tension test on an SHCC specimen. To account for the mismatch in data between the experimental single-sided pullout test and the fully embedded fibers in the SHCC sample, the material models were calibrated based on a quasi-static tension test on the SHCC. The experimental loading conditions during the split Hopkinson bar tension test were replicated using a piece-wise polynomial function. The resulting stress-strain curve of the dynamic simulation showed a significant increase in stress compared to the quasi-static results. This was followed by a thorough parameter study. The two loading parameters were analyzed to show a significant influence on the macroscopic results. This showed that dynamic measurements represent always a structural response and need to be interpreted carefully. One tool to do so is to use the presented framework. The further two parameter studies focused on the microscale. By varying the strain-rate sensitivity of the fibers, the influence of the fibers on the macroscopic response was visualized. By increasing the sensitivity higher stress levels were observed. In addition, another structural effect was visible. The applied loading led to a phase of rigid body motion of the specimen, resulting in a temporary decrease in strain rate. Finally the computation was run without the influence of microscale inertia. As it turned out, the overall macroscopic results were not significantly influenced by microscopic inertia, only high frequency oscillations were observed as change in effective macroscopic stress. This is not surprising, as the simplified microstructure combined with the chosen material models does not allow for significant microscopic inertia effects. The initial findings imply that micro inertia might not have a pronounced direct effect on the resulting macroscopic stress. However, it is likely that more advanced micromechanical models will show a more pronounced indirect effect by directly changing the fiber pullout and the crack evolution. Furthermore, there is the possibility that for microstructures allowing for multiple cracks, the direct effect will increase. More research is needed to give a definite answer. Nevertheless, there are two insights from this parameter study. One, that even small effects as a single crack opening and closing, can be observed at the macroscale. Two, that this type of analysis is only possible by using a two-scale framework that includes the full inertia effects at the microscale. By developing more sophisticated material models while conducting further dynamic micromechanical experiments, the predictive capacity of the simulation can be further improved. For future investigations aiming at a more detailed assessment of the developed RVEs, the micromechanical testing configurations will be adapted for higher displacement rates (such as presented in [32]). This will allow for a more realistic assessment of the dynamic fiber tensile strength and fiber-matrix bond strength. Moreover, the constitutive morphology (e.g. fiber distribution and orientation and flaw size distribution) of SHCC will be modeled based on statistical measures obtained by microtomography scans [33], allowing a more realistic simulation of the micromechanical and statistical influences on the multiple cracking process.

Author Contributions: The contributions of the authors are listed in the categories: conceptualization, E.T., I.C., V.M and D.B.; methodology, E.T.; numerical analysis, E.T.; experimental data, I.C.; data curation, E.T. and I.C.; writing-original draft preparation,E.T.; writing-review and editing, E.T, I.C., V.M. and D.B.; visualization, E.T.; supervision, V.M. and D.B.; project administration, D.B.; funding acquisition, V.M. and D.B. All authors have read and agreed to the published version of the manuscript.

Funding: This research is funded by the Deutsche Forschungsgemeinschaft within the framework of the Research Training Group GRK 2250 "Mineral-bonded composites for enhanced structural impact safety", project number 287321140 .

Acknowledgments: The authors gratefully acknowledge funding by the Deutsche Forschungsgemeinschaft (DFG - German Research Foundation) in the context of the Research Training Group GRK 2250/1 "Mineral-bonded composites for enhanced structural impact safety", project number 287321140. 
Conflicts of Interest: The authors declare no conflict of interest. The funders had no role in the design of the study, in the collection, analyses, or interpretation of data, in the writing of the manuscript, or in the decision to publish the results.

\section{References}

1. Li, V.C. On Engineered Cementitious Composites (ECC) A Review of the Material and Its Applications. Journal of Advanced Concrete Technology 2003, 1, 215 - 230.

2. Li, V.; Leung, K. Steady-state and multiple cracking of short random fiber composties. Journal of Engineering Mechanics 1992, 118, 2246 - 2264.

3. Mechtcherine, V. Novel cement-based composites for the strengthening and repair of concrete structures. Construction and building materials 2013, 41, 365-373. doi:10.1016/j.conbuildmat.2012.11.117.

4. Curosu, I. Influence of fiber type and matrix composition on the tensile behavior of strain-hardening cement-based composites (SHCC) under impact loading. Doctoral Dissertation, Schriftenreihe des Instituts für Baustoffe Heft 2018/1, V. Mechtcherine (Hrsg.), Technische Universität Dresden, 2018. ISBN 978-3-86780-555-1.

5. Curosu, I.; Mechtcherine, V.; Hering, M.; Curbach, M. Mineral-bonded composites for enhanced strucutural impact safety - Overview of the format, goals and achievements of the research training group GRK 2250. 10th International Conference on Fracture Mechanics of Concrete and Concrete Structures 2019. doi:10.21012/FC10.235408.

6. Fish, J.; Chen, W.; Nagai, G. Non-local dispersive model for wave propagation in heterogeneous media: multi-dimensional case. International Journal for Numerical Methods in Engineering 2002, 54, 347-363.

7. Craster, R.V.; Kaplunov, J.; Pichugin, A.V. High-frequency homogenization for periodic media. Proceedings of the Royal Society A 2010, 466, 2341-2362.

8. Hui, T.; Oskay, C. A high order homogenization model for transient dynamics of heterogeneous media including micro-inertia effects. Computer Methods in Applied Mechanics and Engineering 2014, 273, 181-203.

9. Hu, R.; Oskay, C. Multiscale nonlocal effective medium model for in-plane elastic wave dispersion and attenuation in periodic composites. Journal of the Mechanics and Physics of Solids 2019, 124, 220-243.

10. Bensoussan, A.; Lions, J.L.; Papanicolaou, G. Asymptotic analysis for periodic structures; North-Holland Publishing Company: Amsterdam, 1978.

11. Willis, J.R. Dynamics of composites; Springer Vienna, 1997.

12. Milton, G.W.; Willis, J.R. Minimum variational principles for time-harmonic waves in a dissipative medium and associated variational principles of Hashin-Shtrikman type. Proceedings of the Royal Society A: Mathematical, Physical and Engineering Sciences 2010, 466, 3013-3032.

13. Nemat-Nasser, S.; Srivastava, A. Overall dynamic constitutive relations of layered elastic composites. Journal of the Mechanics and Physics of Solids 2011, 59, 1953-1965.

14. Willis, J.R. The construction of effective relations for waves in a composite. Comptes Rendus Mécanique 2012, 340, 181-192.

15. de Souza Neto, E.; Blanco, P.; Sánchez, P.; Feijóo, R. An RVE-based multiscale theory of solids with micro-scale inertia and body force effects. Mechanics of Materials 2015, 80, 136-144.

16. Karamnejad, A.; Sluys, L.J. A dispersive multi-scale crack model for quasi-brittle heterogeneous materials under impact loading. Computer methods in applied mechanics and engineering 2014, 278, 423-444.

17. Liu, C.; Reina, C. Variational coars-graining procedure for dynamic homogenization. Journal of the Mechanics and Physics of Solids 2017, 104, 187-206.

18. Liu, C.; Reina, C. Dynamic homogenization of resonant elastic metamaterials with spacetime modulation. Computational Mechanics 2018, 64, 147-161.

19. Pham, K.; Kouznetsova, V, G.; Geers, M. Transient computational homogenization for heterogeneous materials under dynamic excitation. Journal of the Mechanics and Physics of Solids 2013, 61, 2125-2146.

20. Roca, D.; Lloberas-Valls, O.; Cante, J.; Oliver, J. A computational multiscale homogenization framework accounting for inertia effects: Application to acoustic metamaterials modelling. Computer methods in applied mechanics and engineering 2018, 330, 415-446.

21. Sridhar, A.; Kouznetsova, V.G.; Geers, M.G.D. A general multiscale framework for the emergent effective elastodynamics of metamaterials. Journal of the Mechanics and Physics of Solids 2018, 111, 414-433. 
22. Tamsen, E.; Balzani, D. A general, implicit, large-strain $\mathrm{FE}^{2}$ framework for the simulation of dynamic problems on two scales. Computational Mechanics 2020. In Review.

23. Curosu, I.; Mechtcherine, V.; Millon, O. Effect of fiber properties and matrix composition on the tensile behavior of strain-hardening cement-based composites (SHCCs) subject to impact loading. Cement and Concrete Research 2016, 82, 23 - 35. doi:https://doi.org/10.1016/j.cemconres.2015.12.008.

24. Curosu, I.; Mechtcherine, V.; Forni, D.; Cadoni, E. Performance of various strain-hardening cement-based composites (SHCC) subject to uniaxial impact tensile loading. Cement and Concrete Research 2017, 102, 16-28.

25. Curosu, I.; Liebscher, M.; Mechtcherine, V.; Bellman, C.; Michel, S. Tensile behavior of high-strength strain hardening cement based composites (HS-SHCC) made with high-performance polyethylene, aramid and PBO fibers. Cement and Concrete Research 2017, 102, 16- 28.

26. Heravi, A.A.; Curosu, I.; Mechtcherine, V. A gravity-driven split Hopkinson tension bar for investigating quasi-ductile and strain-hardening ce-ment-based composites under tensile impact loading. Cement and Concrete Composites 2020, 105, 103430.

27. Blanco, P.; Sánchez, P.; de Souza Neto, E.; Feijóo, R. Variational Foundations and Generalized Unified Theory of RVE-Based Multiscale Models. Archives of Computational Methods in Engineering 2016, 23, 191-253.

28. Newmark, N.M. A method of computation for structural dynamics. Journal of Engineering Mechanics 1959, 85, 67-94.

29. Tamsen, E.; Weber, W.; Balzani, D. First steps towards the direct micro-macro simulation of reinforced concrete under impact loading. Proceedings in Applied Mathematics and Mechanics 2018, 18, e201800181.

30. Bonet, J.; Wood, R.D. Nonlinear Continuum Mechanics for Finite Element Analysis, 2 ed.; Cambridge University Press, 2008. doi:10.1017/CBO9780511755446.

31. Kolsky, H. An investigation of the mechanical properties of materials at very high rates of loading. Proceedings of the Physical Society Section B 1949, 62, 676- 700.

32. Trindade, A.C.C.; Heravi, A.A.; Curosu, I.; Liebscher, M.; Mechtcherine, V. Tensile behavior of strain-hardening geopolymer composites (SHGC) under impact loading. Cement and Concrete Composites 2020, 113, 103709.

33. Lorenzoni, R.; Curosu, I.; Léonard, F.; Paciornik, S.; Mechtcherine, V.; Silva, F.A.; Bruno, G. Combined mechanical and 3D-microstructural analysis of strain- hardening cement-based composites (SHCC) by in situ X-ray microtomography. Cement and Concrete Research 2020, 136, 106139. 